\title{
Macrophage Phenotypes and Hepatitis B Virus Infection
}

\author{
Yujia $\mathrm{Li}^{1}$, Shilin $\mathrm{Li}^{1}$, Xiaoqiong Duan ${ }^{1}$, Chunhui Yang ${ }^{1}$, Min $\mathrm{Xu}^{1}$ and Limin Chen ${ }^{1,2 *}$ \\ ${ }^{1}$ Institute of Blood Transfusion, Chinese Academy of Medical Sciences and Peking Union Medical College, Chengdu, Sichuan, \\ China; ${ }^{2}$ Toronto General Research Institute, University of Toronto, Toronto, ON M5G 1L6, Canada
}

\begin{abstract}
Globally, hepatitis B virus (HBV) infection and its related liver diseases account for 780,000 deaths every year. Outcomes of $\mathrm{HBV}$ infection depend on the interaction between the virus and host immune system. It is becoming increasingly apparent that Kupffer cells (KCs), the largest population of resident and monocyte-derived macrophages in the liver, contribute to HBV infection in various aspects. These cells play an important role not only in the anti-HBV immunity including virus recognition, cytokine production to directly inhibit viral replication and recruitment and activation of other immune cells involved in virus clearance but also in HBV outcome and progression, such as persistent infection and development of end-stage liver diseases. Since liver macrophages play multiple roles in HBV infection, they are directly targeted by HBV to benefit its life cycle. In the present review, we briefly outline the current advances of research of macrophages, especially the studies of their phenotypes, in chronic HBV infection.
\end{abstract}

Citation of this article: Li Y, Li S, Duan X, Yang C, Xu M, Chen L. Macrophage phenotypes and hepatitis $B$ virus infection. J Clin Transl Hepatol 2020;8(4):424-431. doi: 10.14218/ JCTH.2020.00046.

\section{Introduction}

As a major world health problem, hepatitis B virus (HBV) infects 257 million people, representing about $3 \%$ of the

Keywords: Hepatitis B virus; Chronic HBV infection; Macrophage; Phenotype. Abbreviations: ALT, alanine aminotransferase; APC, antigen-presenting cell ATP, adenosine triphosphate; $\mathrm{CHB}$, chronic HBV infection; $\mathrm{CHC}$, chronic hepatitis $C$ virus; $C T L$, cytotoxic $T$ lymphocyte; $C X C L$, chemokine (C-C motif) ligand, $C C L$, $\mathrm{C}-\mathrm{X}-\mathrm{C}$ motif chemokine ligand; DC, dendritic cell; GM-CSF, granulocyte-macrophage colony-stimulating factor; HBV, hepatitis B virus; $\mathrm{HCC}$, hepatocellular carcinoma; HCV, hepatitis C virus; HSPG, heparan sulfate proteoglycan; IFN, interferon; IL, interleukin; IRF3, interferon regulatory factor 3; ISGs, IFN-stimulated genes; KC, Kupffer cell; LBP, LPS binding protein; LCMV, lymphocytic choriomeningitis virus; $L P L$, lipoprotein lipase; LSEC, liver sinusoidal endothelial cell; M-CSF, macrophage-colony stimulating factor; MDM, monocyte-derived macrophage; MHC, major histocompatibility complex; MHV, murine hepatitis virus; $M I F$, macrophage migration inhibitory factor; $M R$, mannose receptor; $M \times A$, myxovirus resistance gene; NF-кB, nuclear factor kappa-B; NK, natural killer; NKT, natural killer T; NTCP, sodium taurocholate cotransporting polypeptide; OXPHOS, oxidative phosphorylation; PBMC, peripheral blood mononuclear cell; PEM, peritoneal exudative macrophage; PGE2, prostaglandin E2; $\mathrm{PTH}$, primary tupaia hepatocytes; ROS, reactive oxygen species; STING, stimulator of IFN genes; TLR, toll-like receptor; TNF, tumor necrosis factor; TRAIL, TNF-related apoptosis-inducing ligand.

Received: 14 May 2020; Revised: 20 August 2020; Accepted: 6 September 2020 * Correspondence to: Limin Chen, Institute of Blood Transfusion, Chinese Academy of Medical Sciences, Peking Union Medical College, 26 Huacai Street, Chengdu, Sichuan 610052, China. Tel: +86-28-61648530, E-mail: limin_chen_99@126.com or limin.chen@ibt.pumc.edu.cn world's population (https://www.who.int/en/news-room/ fact-sheets/detail/hepatitis-b). While approximately $95 \%$ of HBV infections acquired during adulthood are resolved, the virus cannot be cleared in most individuals infected in perinatal period or early childhood. ${ }^{1}$ It is widely accepted that the virus-host interaction, which is affected by age, transmission route, immune status and other factors, determines the outcome of infection. ${ }^{2,3}$

Both adaptive and innate immunity are involved in antiHBV immune response. On one hand, antigen-presenting cells (APCs), including macrophages and dendritic cells (DCs), initialize the virus-specific adaptive immunity characterized by activation of T helper lymphocytes and secretion of various cytokines, which then mobilize the cytotoxic $T$ lymphocyte (CTL) to kill the HBV-infected cells. Additionally, HBVspecific antibodies are developed by the humoral immune system to neutralize the virus and facilitate its clearance. ${ }^{4}$ On the other hand, the essential role of non-specific defense, especially the function of the liver macrophages (i.e. KCs), has gained growing attention (reviewed in Faure-Dupuy et $a l .{ }^{5}$ ), albeit the precise mechanism remains incompletely elucidated because of the difficulty in identifying asymptomatic early infections in human studies. ${ }^{6}$ Unlike hepatitis $C$ virus (HCV), HBV was once considered as a "stealth virus", due to the fact that HBV could not induce significant innate immune response in an acute HBV-infected chimpanzee model. $^{7}$ Limited evidence from clinical study ${ }^{8}$ also showed that no intense cytokine storm, such as type I interferon (IFN) and type III IFN production, occurs in patients with acute HBV infection. Nevertheless, one typical characteristic of HBV infection is macrophage hyperplasia in the liver, ${ }^{9,10}$ suggesting an important role of macrophages in HBV pathogenesis. It has been demonstrated that some effecter molecules, such as interleukin (IL)- $6,{ }^{11}$ were produced by KCs to replace IFNs to control HBV infection. ${ }^{11}$ Another interesting study ${ }^{12}$ showed that HBV DNA in the liver and blood were cleared before the adaptive immune response was elicited, indicating that innate immune response is much more than a simple branch to control virus invasion until onset of the adaptive response.

Herein, we will review the effects of liver macrophages on $\mathrm{HBV}$ infection, focusing on macrophage phenotypes in HBV persistent infections.

\section{Macrophages: Functions and phenotypes}

In the healthy liver, the compartment of liver macrophages is dichotomic, involving tissue-resident macrophages (i.e. KCs) and monocyte-derived macrophages (MDMs). KCs, as well as the liver DCs and sinusoidal endothelial cells (LSECs), are mainly localized in the sinusoids of the liver and they form the 
first line of defense to diverse antigens and toxic components contained in portal venous blood. ${ }^{13}$ MDMs are mainly localized near the portal triad. When the KCs are depleted experimentally or pathologically, the MDMs can be infiltrated from the peritoneal cavity, replacing KCs by acquiring virtually the same phenotype. ${ }^{14}$ In fact, the liver macrophages (i.e. KCs and MDMs) are very plastic and no specific marker is used to discriminate KCs from MDMs in human. ${ }^{5}$

As an important component of innate immunity, liver macrophages can function as: 1) phagocytes to remove dead cells, debris and pathogens; ${ }^{15}$ 2) effective APCs, with their expression of major histocompatibility complex (MHC) and co-stimulatory molecules; 3 ) immune mediators involved in immune suppression and allograft tolerance of liver; and, 4) key players in rapid erythrocyte removal and iron recycling (mainly the MDMs). ${ }^{16}$

Polarization, which is equally vague as activation, is indispensable before the macrophages achieve their different functions. Generally speaking, macrophages can be polarized into two major subsets with different combinations of stimuli: ${ }^{17}$ M1 macrophages of classical activation, which induce inflammation and cause tissue damage by facilitating Th1 response, and M2 macrophages of alternative activation, which maintain tissue integrity by promoting the Type $2 \mathrm{~T}$ helper cell response (Fig. 1). M1 and M2 type cytokines or surface markers are referred to, to differentiate different macrophage activation phenotypes. There are other subsets, such as M2a, M2b, M2c, etc. What has been overlooked, however, is that polarization is a process which changes continuously ${ }^{18}$ and various mixtures of M1 and M2 type macrophages may result in confusion. As a matter of fact, much more effort is required to define the criteria for assessing phenotypes. However, for the rest of this review, we will discuss the association between macrophages and HBV infection on the basis of the current understanding of M1/M2 type macrophages.

\section{Do liver macrophages sense HBV infection?}

First of all, although the exact interaction between liver macrophages and HBV is still unclear, nonhepatic cell surface presentations of molecules interacting with Pres or hepatitis B core antigen have been documented. Peripheral blood mononuclear cells (PBMCs), the monocytic cell line THP-1 and U937 ${ }^{19-23}$ were reported to express the PreSbinding receptor of HBV. Lipoprotein lipase (LPL), which can be produced by THP-1 macrophages, ${ }^{24,25}$ has an linear motif for Pres binding and may interact with HBV particles during infection. ${ }^{23}$ Additional candidate Pre-S receptors, which can be expressed by $\mathrm{KCs}^{26}$ are lipopolysaccharide (LPS) binding protein (LBP), the LPS receptor CD14, ${ }^{22}$ and mannose receptor (MR). ${ }^{27,28}$ These receptors are involved in the binding of hepatitis $B$ surface antigen to macrophages, monocytes or DCs. Hepatitis B core antigen was also reported to bind to PBMCs and trigger the release of IL-18. ${ }^{29}$ Consistently, Cooper et al. ${ }^{30}$ demonstrated that hepatitis B core antigen could bind to receptor(s), like the Toll-like receptor (TLR)2 and heparan sulfate proteoglycan (HSPG), on THP-1 macrophages by its arginine-rich domain at the C-terminal and effectively induce expression of pro-inflammatory molecules. Given the fact that hepatitis B core antigen mainly exists within the hepatocytes and viral particles, whether liver macrophages interact with hepatitis $B$ core antigen during HBV infection in patients is still not clear. Accordingly, although there is a probable involvement of HBV antigen receptors in initializing viral infection, it is more likely that these receptors only mediate cellular recognition or internalization of HBV/ HBV antigens. Little work has addressed the expression of the recently identified HBV functional receptor sodium taurocholate cotransporting polypeptide (NTCP) ${ }^{31,32}$ in liver macrophages. In an interesting study, Neurath et al. ${ }^{20}$ reported that HUT-78 and MOLT3 cells (both T cell lines) could covalently attach to PreS-cellulose or hepatitis B surface antigencellulose after treatment with concanavalin A linked with a peptide of HBV PreS1. This result suggests a similar possibility that HBV receptors could be induced by appropriate stimulations in liver macrophages or monocytes. Moreover, HBV antigens and nucleic acid have been detected in macrophages and monocytes, ${ }^{21,33-35}$ raising the possibility that HBV might be "taken into" the macrophages or monocytes.

Secondly, the ability of macrophages to produce cytokines upon exposure to HBV potentially renders them as indispensable immune cells sensing and discriminating invading HBV. Hösel's group11 observed an early-time, nuclear factor kappa-B (NF-кB)-dependent induction of inflammatory mediators in primary human KCs stimulated with HBV inoculum generated from the HepG2.2.15 cell line. This cluster of soluble inflammatory cytokines, including IL-6, IL-8, IL-1 $\beta$ and tumor necrosis factor (TNF)- $\alpha$ but no type I IFN, inhibited $\mathrm{HBV}$ replication significantly. In a more recent study, ${ }^{21} \mathrm{KCs}$ isolated from patients with persistent HBV infection showed a higher activation status (characterized by elevated expressions of CD40, HLA-ABC and HLA-DR) than those of healthy control. And, in accordance with previous report, their experiments ${ }^{21}$ also revealed obvious inductions of IL-6, IL-15, TNF, chemokine ( $\mathrm{C}-\mathrm{C}$ motif) ligand 4 (CCL4), C-X-C motif chemokine ligand 8 (CXCL8), as well as IL-10 in human primary KCs and PBMC-generated macrophages cultured with patient plasma-derived hepatitis B surface antigen. Most recently,

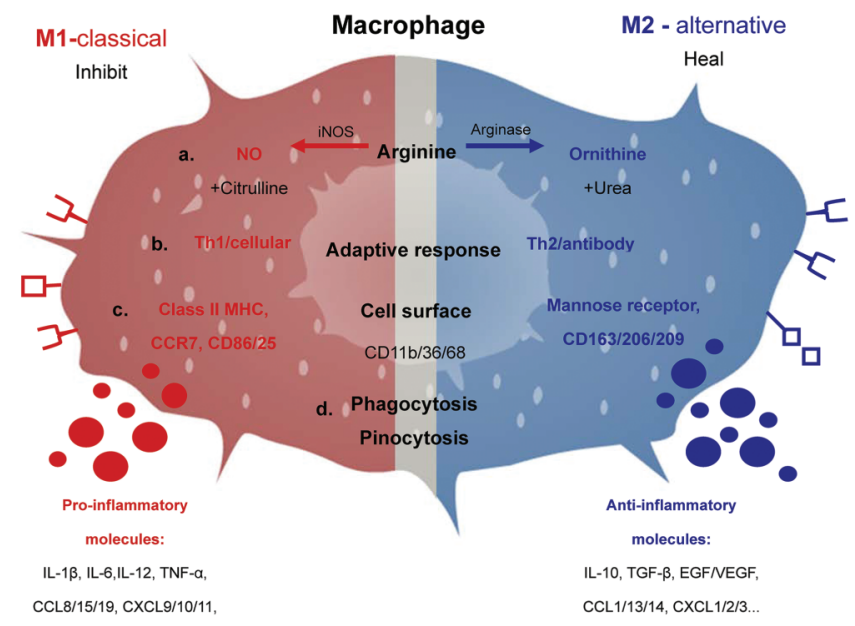

Fig. 1. Characteristic products and functions of M1 and M2 macrophages. Macrophages can metabolize arginine with the inducible nitric oxide synthase enzyme into nitric oxide and citrulline or with arginase into ornithine and urea which is the biochemical basis of the M1 or M2 macrophage responses, respectively (a). M1- or M2-dominant macrophages stimulate the Type $1 \mathrm{~T}$ helper cell or Type $2 \mathrm{~T}$ helper cell responses (b). Also shown are the major molecules involved, including cell surface molecules, cytokines, chemokines and so on, which are closely associated with the M1 or M2 phenotypes (c). Phagocytosis and pinocytosis are general properties of macrophages, which are not dependent on M1 or M2 type responses (d). 
Cheng et al. ${ }^{36}$ reported that human macrophages showed an inflammatory cytokine storm when stimulated with high level $\mathrm{HBV}$, while the hepatocytes sensed HBV DNA poorly. Other in vitro studies demonstrated that HBV antigens (e.g., HBV envelop protein, PreS and HBV core antigen) were able to induce cytokine secretion in monocytes and MDMs after binding to the receptors (see below).

\section{M1 and M2 macrophages involved in HBV infection}

As a major source of cytokines and immune regulators, macrophages are involved in HBV infection in at least two aspects: (1) antiviral effects, mainly mediated by M1 type molecules (Fig. 2a); and, (2) immunotolerance, mediated by M2 type molecules (Fig. 2b).

\section{Antiviral effects}

Activation of M1-type macrophages and production of proinflammatory cytokines usually indicate a robust immune response to HBV infection. CD16+ is one of the M1-like phenotype markers. Zhang's group ${ }^{37}$ investigated 110 hepatitis $B$ e antigen-negative chronic hepatitis B ( $\mathrm{CHB}$ ) patients and found that the immune-activated group was characterized by lower HBV DNA and that high alanine aminotransferase (ALT) is associated with more CD16+ monocytes and/or macrophages in the peripheral blood and liver, when compared with the immune tolerant group. High level of M1-like CD16+ macrophages was an indicator for immune activation that helped patients to defend against the virus.

Direct antiviral effects: Agonists of TLRs ${ }^{38-41}$ and HBV antigens (as discussed above) can induce macrophages to express soluble inflammatory mediators and other effective molecules, which are the major effectors to assume direct antiviral activity of macrophages. With diverse mechanisms, these effective molecules can either control HBV without obvious cytotoxicity or result in injury or apoptosis of the infected hepatocytes.

Type I IFN, for example, one of the key cytokines potently inhibiting HBV replication in hepatocytes, is routinely used in the clinic to treat HBV patients. Despite plenty of work having addressed the anti-HBV mechanisms of type I IFN, there is limited clinical or in vivo evidence for the idea that after the lag phase of HBV replication with negligible release of type I IFN, liver macrophage-synthesized IFN $\alpha / \beta$ may act as essential controller for HBV. Fortunately, circumstantial evidence is

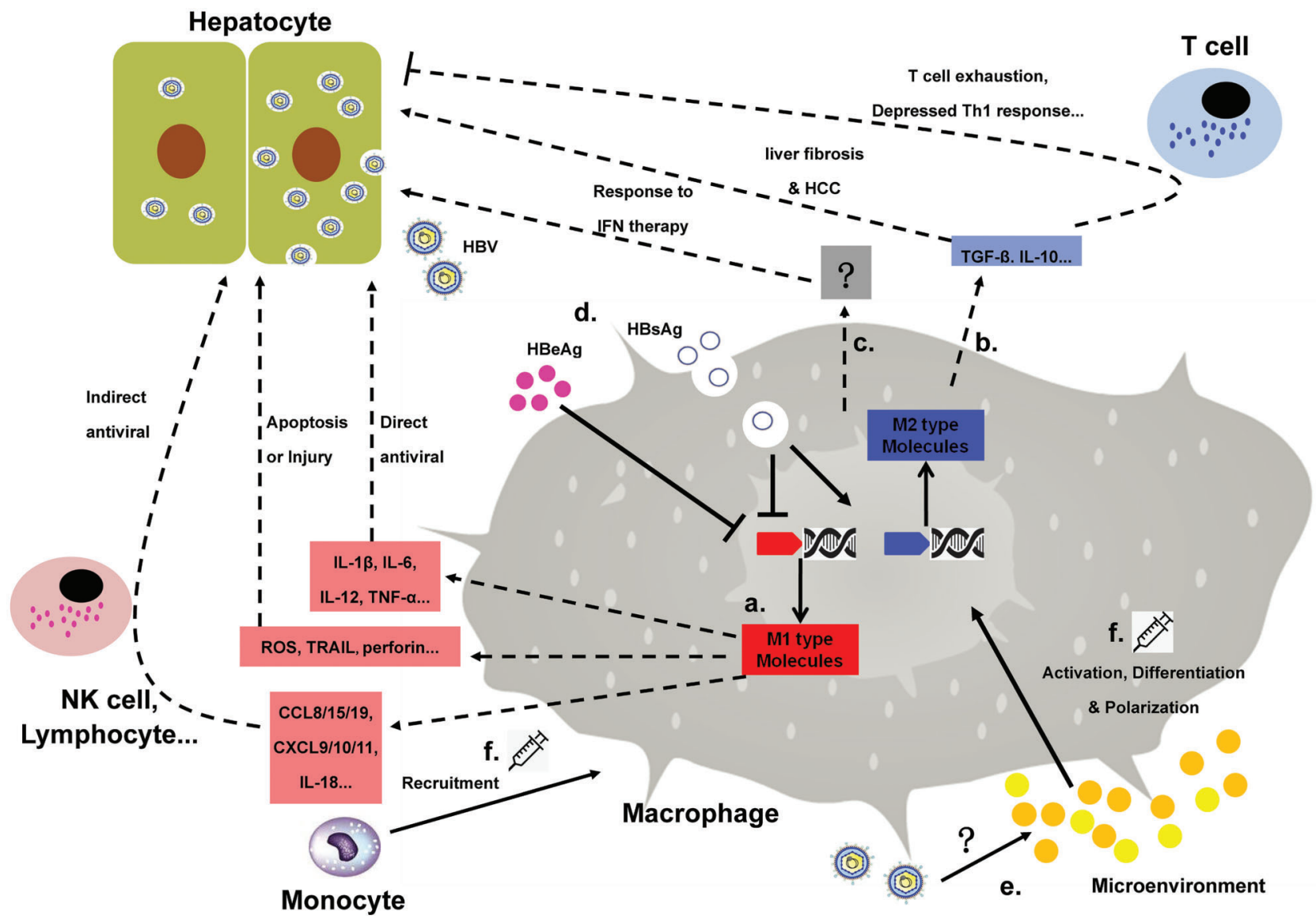

Fig. 2. Macrophage involvement in HBV infection. The anti-HBV effect of macrophages is mediated mainly by pro-inflammatory cytokines inducing a direct antiviral response or molecules recruiting or activating other immune cells. Meanwhile, another group of M1 KCs produces molecules that may result in injury or apoptosis of the hepatocytes (a). Immunomodulatory mediators, such as IL-10 and TGF- $\beta$, are closely associated with suppressed antiviral T cell responses and/or end-stage HBV liver disease (b). Macrophages may also contribute to the inflammatory or anti-inflammatory liver microenvironment and, consequently, alter hepatic response to IFN treatment (c). The phenotype and function of macrophages can be modified by either HBV itself (d) or the microenvironment (e). Thus, the therapeutic strategies targeting macrophages in an HBV infection may aim at modulating macrophage polarization/phenotype, monocyte recruitment/activation and so on (f). 
available. First of all, type I IFNs induced by in vitro activated KCs effectively suppress HBV production. Injections of diverse TLR (TLR3/4/5/7/9) agonists could control HBV replication, which is IFN $\alpha / \beta$-dependent in transgenic mice. ${ }^{40}$ Wu's group ${ }^{38}$ confirmed and extended these findings by collecting the supernatants of primary C57BL/ 6 mouse KCs after stimulation with ligands specific for TLR1 to TLR9 and evaluating their effects on HBV-Met cells. Their study showed a significant TLR3- or TLR4-mediated suppression of HBV replication, which can be abolished, or at least partially abolished, by IFN$\beta$ antibodies.

Another study ${ }^{42}$ using KCs activated by the agonist of stimulator of IFN genes (STING) revealed a predominant type I IFN production and subsequent inhibition of HBV replication in the AML12HBV10 cell line. The anti-HBV effect of STING agonist was further confirmed in the HBV DNA hydrodynamic NOD/SCID mouse model. ${ }^{42}$ Secondly, unrelated viral infection may activate $\mathrm{KCs}$ and noncytopathically inhibit HBV production via IFN $\alpha / \beta$. Guidotti et al. ${ }^{43}$ used lymphocytic choriomeningitis virus (LCMV) to infect HBV transgenic mice and assayed the production of HBV. They found that 3.5- and 2.1-kb HBV mRNAs were decreased or even absent, as well as HBV DNA replication forms. Similar results were found in HBV transgenic mouse model with malaria infection. ${ }^{44}$ Recruitment of macrophages and subsequently elevated expressions of IFN $\alpha / \beta / \gamma$ suppressed HBV gene expression and replication in vivo. Thirdly, HBV has evolved some strategies specifically targeting IFN production in KCs, indicating the potential antiHBV effects of macrophage-derived IFNs. Using the murine nonparenchymal liver cells, Wu's group ${ }^{45}$ demonstrated that hepatitis $b$ surface antigen, hepatitis $B$ e antigen, as well as HBV virion, could suppress TLR3-mediated IFN- $\beta$, IFN- $\gamma$ and IFN-stimulated gene (ISG) production by interfering with the activation of interferon regulatory factor 3 and NF-kB.

Activated macrophages are the major source of TNF- $\alpha,{ }^{46}$ which has been identified as a potent anti-HBV molecule. It has been well established that TNF- $\alpha$ production increases in the primary $\mathrm{KCs}^{47}$ or $\mathrm{PBMCs}^{48}$ isolated from $\mathrm{CHB}$ patients. In vitro studies also demonstrated that the expression levels of TNF- $\alpha$ in primary KCs, ${ }^{11,21} \mathrm{MDMs}^{21}$ and THP- ${ }^{30}$ cells were up-regulated in response to HBV challenge. In addition, HBV replication in primary tupaia hepatocytes (PTHs) was partially inhibited by recombinant tupaia TNF- $\alpha .{ }^{49}$ In transgenic mice, TNF- $\alpha$ produced by macrophages during LCMV, ${ }^{43}$ adenovirus or cytomegalovirus ${ }^{50}$ infection inhibited HBV gene expression and DNA replication noncytopathically. Furthermore, substantial clinical data also raised the importance of TNF- $\alpha$ in HBV infection.

Elimination of hepatitis B e antigen and suppression of $\mathrm{HBV}$ replication in patients receiving IFN $\alpha$ treatment was accompanied by spontaneously induced TNF- $\alpha$ in PBMCs. ${ }^{51}$ Anti-TNF- $\alpha$ therapy in patients with chronic inflammatory diseases was associated with higher risk of HBV activation, reactivation and hepatotoxicity, ${ }^{52-55}$ which may be attributed to the setting of immune suppression. Accumulating evidence also suggests an important role of TNF- $\alpha$ gene polymorphisms in HBV infection. ${ }^{56}$ These clinical studies, together with the data from basic research and animal models, mirror the fact that TNF- $\alpha$, as well as the macrophages, is one of the prerequisites for virus clearance and permanent control of HBV.

Other macrophage-derived anti-HBV cytokines include IL$1 \beta,{ }^{11,51} \mathrm{IL}-6,{ }^{11,21,30} \mathrm{IL}-12,{ }^{57} \mathrm{IL}-15,{ }^{21}$ and macrophage migration inhibitory factor (MIF), ${ }^{58}$ some of which may perform synergistic actions with each other. ${ }^{59}$ Meanwhile, another group of KCs produced molecules, such as reactive oxygen species (ROS), ${ }^{10}$ Fas-ligand, ${ }^{60}$ TNF-related apoptosis-inducing ligand (TRAIL), ${ }^{61}$ granzyme $B$ and perforin, ${ }^{62}$ may result in injury or apoptosis of the hepatocytes.

Indirect antiviral effects through recruiting or activating other immune cells: Liver macrophages synthesize several cytokines and chemokines to activate or recruit inflammatory cells involved in the anti-HBV roles. IL-18, an inflammatory cytokine belonging to the IL-1 family, is mainly expressed by liver macrophages. ${ }^{63}$ Previous studies have demonstrated that IL-18 plays a powerful anti-HBV role by inducing cytokine production (e.g. IFN- $\gamma$, IFN $\alpha / \beta$, TNF- $\alpha)^{64}$ in some immune cells. Kakimi et al. ${ }^{65}$ showed that IL-18 was a type I- and type II-IFN inducing factor, acting on both intrahepatic natural killer (NK) and natural killer T (NKT) cells in a transgenic mouse model, resulting in suppressed HBV replication. Interestingly, this inhibitory effect of IL-18 on HBV replication is dependent on IL-12, which is able to be released by activated macrophages. Boltjes et al. ${ }^{21}$ analyzed the function of human primary KCs and in vitro-generated MDMs. They found both could be activated by exposure to patient-derived hepatitis B surface antigen, resulting in activation of NK cells characterized by up-regulation of CD69 and IFN- $\gamma$.

It has been well established that Type $1 \mathrm{~T}$ helper cells, B cells and DCs can also produce IFN- $\gamma$ in response to IL-18 stimulation. ${ }^{66-68}$ In addition, activated liver macrophages also produce $\mathrm{CXCL}, \mathrm{CXCL}-9$ and $\mathrm{CXCL}-10$, which assist in trafficking of lymphocytes and monocyte/macrophages into the tissue. ${ }^{69,70}$ Another study from Kakimi group ${ }^{71}$ demonstrated that CXCL-9 and CXCL-10 derived from nonparenchymal cells (including KCs) chemoattracted lymphomononuclear inflammatory cells into the liver in a transgenic murine model.

\section{Immunotolerance/immunosuppressive activity}

Constantly exposed to diverse antigens derived from food or microbial products, the immune cells, in addition to other cells $^{72}$ in the liver, develop some mechanisms to prevent excessive activation and continuous pathology, known as inherent tolerogenicity of the liver. KCs are involved in the well-known tolerogenic milieu by secreting soluble immunoregulators (e.g., IL-10, TGF- $\beta$, and amphiregulin) or expressing inhibitory molecules on the membrane, both of which could be exploited by HBV for their favorable immunosuppressive microenvironment.

For instance, IL-10 could depress inflammation response by inhibiting Type $1 \mathrm{~T}$ helper cell cytokine expression. An HBVcarrier mouse model showed no significant immune response to hepatitis $B$ surface antigen vaccination, which could be reversed by KC depletion or IL-10 deficiency. ${ }^{73}$ Clinical data ${ }^{74}$ also revealed an association between $\mathrm{CHB}$ and elevated plasma IL-10 level, though it was uncertain whether the increased IL-10 was derived mainly from macrophages or not. Consistently, Li's group ${ }^{75}$ also reported that the increased production of IL-10 by KCs, which was stimulated by HBV core antigen, resulted in inhibition of the antiviral function of CD8+ T cells in mice.

Interestingly, IL-10 gene promoter polymorphisms were reported to be associated with HBV progression. ${ }^{76}$ It was reported that murine KCs preferred to produce TGF- $\beta$, which is able to restrain immune response and to develop tolerance towards self-antigens, ${ }^{77}$ rather than functioning as a 
pro-inflammatory cytokine in response to HBV infection. ${ }^{78}$ Although the precise mechanism remains unclear, the tolerogenic role of HBV by modulating liver macrophage polarization should not be ignored, since IL-10 and TGF- $\beta$ are typical cytokines of M2 macrophages. Previous studies also suggested that KCs were primarily immunosuppressive, mediated by prostaglandin E2 (PGE2); ${ }^{13}$ accordingly, HBV may maintain or even promote this immunosuppressive status by regulating macrophage polarization and/or PGE2 production to benefit its replication.

Bility and colleagues ${ }^{79}$ found that $\mathrm{CHB}$ patients with fibrosis and/or hepatocellular carcinoma ( $\mathrm{HCC}$ ) and patients with acute HBV-associated liver failure experienced a M2 phenotype, including increased M2 macrophages in the liver infiltration and predominate M2-type gene expression profile in the liver. The authors developed a humanized mouse model, supporting HBV replication to investigate the HBV-associated immunopathogenesis. They found that impaired immune response in parallel with robust M2-type macrophage activation in the liver contributed to the development of persistent HBV infection, indicating the M2 macrophages might act as immune suppressors. Nowadays, accumulating evidence indicates that macrophages play an important role in HBVinduced immune suppression, not only in persistent infection establishment but also in the development of the end-stage liver diseases, such as liver fibrosis and HCC. ${ }^{80}$

\section{Potential role of macrophages in response to IFN $\alpha$ treatment in $\mathrm{CHB}$ patients}

Type I IFN is still one of the most important therapies for $\mathrm{CHB}$ infection or chronic hepatitis $\mathrm{C}$ virus ( $\mathrm{CHC}$ ). However, only a subset of the patients respond. Our previous studies showed that cell-type specific ISGs' expression in the liver predicts whether a given patient will respond to IFN treatment among $\mathrm{CHC}^{81}$ or $\mathrm{CHB}^{82}$ patients. We analyzed the pre-treatment gene expression in $38 \mathrm{CHB}$ livers by immunohistochemical staining and found that in the treatment responders, increased ISG15 and myxovirus resistance gene (MXA) protein expression was more pronounced in macrophages than that in hepatocytes. In contrast, in the non-responders, elevated expression of ISG15 and MxA was more pronounced in hepatocytes compared with that in macrophages. A similar result was found in $\mathrm{CHC}$ patients before receiving pegylatedIFN/ribavirin treatment, indicating that the liver macrophages might be involved in mediating patients' response to IFN and other anti-viral therapy.

Many studies correlated IFN and a subset of typical ISGs (e.g., ISG15, USP18) with macrophage phenotypes and functions. Fleetwood et al. ${ }^{83}$ reported that the type I IFN signaling pathway played an essential role in regulating phenotype and function of macrophage-colony stimulating factor (M-CSF)- or granulocyte-macrophage colony-stimulating factor (GM-CSF)-treated bone marrow-derived macrophages in mice. ISG15, a typical ISG, may play an important role in macrophage polarization and function. Macrophage polarization is characterized by mitochondrial functions regulated by different metabolic patterns, and the lack of ISG15 was responsible for mitochondrial dysfunction, including diminished oxidative phosphorylation (OXPHOS), depressed oxygen consumption rate, as well as reduced adenosine triphosphate (ATP) and ROS production in bone marrow-derived macrophages in mice. ${ }^{84}$ Macrophages from the ISG15-deficient mice have been shown to have depressed phagocytic capacity, which is dependent on protein kinase AKT. ${ }^{85}$ Moreover, we previously described USP18, another typical ISG, as a modulator of macrophage activity in mice. Compared with wild type control, both primary KCs or peritoneal exudative macrophages (PEMs) from USP18-/- preferred to polarize to the M2-like phenotype, producing more anti-inflammatory cytokines (e.g., IL-10 and IL-4) and less inflammatory cytokines (e.g., TNF- $\alpha$ and IL-12) in response to murine hepatitis virus (MHV)-3 infection (unpublished data).

The inflammation microenvironment may be changed by macrophages, influencing the response of hepatocytes to IFN treatment. Our previous study ${ }^{86}$ found that pre-treatment with TNF- $\alpha$ or LPS led to an IFN $\alpha$ refractory state in human hepatoma cells and primary murine hepatocytes. We have also investigated the response of primary murine hepatocytes to IFN $\alpha$ after co-culturing with the primary murine hepatocytes and the primary murine USP18-/- (M2 like) or wide type (M1-like) PEMs, using the Transwell co-culture system. We found that hepatocytes co-cultured with USP18-/- PEMs experienced much higher expression of ISGs (including of ISG15, USP18 and MxA) with IFN $\alpha$ stimulation (unpublished data).

We therefore hypothesize that liver macrophages regulate inflammatory and anti- inflammatory responses, contributing to the liver microenvironment and, consequently, alter hepatic response to IFN treatment. However, more in-depth investigations are needed to uncover the underlying molecular mechanism (Fig. 2c).

\section{Effect of HBV on macrophage phenotype}

The distinct roles of M1 and M2 macrophages involved in HBV infection raise the possibility that HBV may promote M2 polarization of macrophages to impair the Type $1 \mathrm{~T}$ helper cell immune response, resulting in persistent infection and disease progression. A most recent study ${ }^{87}$ supported the hypothesis that HBV suppresses M1 macrophage cytokine (IL-6 and IL-1 $\beta$ ) expression and promotes M2 macrophage cytokine (IL-10) expression to favor HBV infection. Although the precise mechanism remains unclear, several in vitro studies have revealed that either hepatitis B surface antigen or hepatitis $B$ e antigen may make a contribution. Expression of M1-type cytokines, such as TNF- $\alpha$, IL- 1 b and IL- 8 , was inhibited by hepatitis $B$ surface antigen in PBMCs, ${ }^{19}$ while the expression of IL-10 was not affected or even promoted. ${ }^{19,88}$ Similar results were observed in THP-1-derived macrophages: hepatitis $B$ surface antigen acted as a potent suppressor of M1-type cytokines, including IL-12, TNF- $\alpha$, IL$1 \beta$ and IL-6. ${ }^{88-90}$ Moreover, Yu et al. ${ }^{91}$ reported a decreased $\mathrm{IL}-1 \beta$ secretion in liver macrophages induced by hepatitis $B$ e antigen. However, it has been reported that pro-inflammatory macrophages and monocytes expressing TNF- $\alpha$ and GM-CSF accumulated in chronic HBV or HCV-related liver disease. ${ }^{92}$ Moreover, high level of IL-23 (as well as IL-1 $\beta$, IL-6 and IL17) expression in liver inflammatory macrophages was demonstrated to be associated with HCC development. ${ }^{93}$ These contradictory lines of evidence have indicated the complicated association between different macrophage phenotypes and disease progression of hepatitis $B$ virus infection (Fig. 2d). In addition, the microenvironment altered by HBV infection may also contribute to the activation, differentiation and polarization of macrophages (Fig. 2e). 


\section{Conclusions}

Although our knowledge about the exact immunological pathogenesis during chronic HBV infection is limited, the remarkable heterogeneity of liver macrophages concerning not only the defense but also the homeostasis and metabolism make it a promising option for treating HBV infection ${ }^{14}$ (Fig. 2f). Binding and/or up-taking virus/ viral antigens, as well as the signaling from infected hepatocytes, may result in the activation of liver macrophages. The activated liver macrophages, on one hand, enforce virus clearance by producing pro-inflammatory cytokines targeting hepatocytes to suppress HBV directly or recruiting, interacting or activating other immune cells to get rid of the virus, and on the other hand, modulating immunotolerance as a negative feedback to avoid unchecked inflammation. This functional diversity or contrary action makes it possible that liver macrophages may be exploited by HBV.

HBV is a stealth virus which "hides" itself in the early stage of life cycle to escape macrophage defenses, and may then manipulate the polarization/phenotype of macrophages to benefit its persistent infection. It is important to note that the response of liver macrophages to HBV infection is not only limited to innate defense but also links the innate immunity with acquired immunity. However, whether macrophages resolve virus, worsen liver immunopathogenesis, promote persistent infection or modulate the response to IFN $\alpha$ therapy depends on a combination of various factors and is finely tuned. This is indeed a complicated process and the interaction between macrophages and HBV deserves further study.

\section{Funding}

Some of the studies summarized in this review were financially supported by grants from the Chinese Academy of Medical Sciences (CAMS) Initiative for Innovative Medicine (CAMS-2016-I2M-3-025 and CAMS-2017-I2M-B\&R-15), the National Key Research Development Program of China (2018YFE0107500 to Professor Limin Chen), and the Science \& Technology Department of Sichuan Province (2020YFH0070 to Yujia Li).

\section{Conflict of interest}

The authors have no conflict of interests related to this publication.

\section{Author contributions}

Contributed to original draft writing $(\mathrm{YL})$, review \& editing (SL, $\mathrm{XD})$, literature research (CY, MX), and conceptualization and funding acquisition (LC).

\section{References}

[1] Aspinall EJ, Hawkins G, Fraser A, Hutchinson SJ, Goldberg D. Hepatitis B prevention, diagnosis, treatment and care: a review. Occup Med (Lond) 2011;61:531-540. doi: 10.1093/occmed/kqr136.

[2] Publicover J, Jespersen JM, Johnson AJ, Nishimura SL, Goodsell A, Wakil AE, et al. Liver capsule: Age-influenced hepatic immune priming determines HBV infection fate: Implications from mouse to man. Hepatology 2016;63:260. doi: $10.1002 /$ hep. 28284 .
[3] Stevens CE, Beasley RP, Tsui J, Lee WC. Vertical transmission of hepatitis B antigen in Taiwan. N Engl J Med 1975;292:771-774. doi: 10. 1056/NEJM197504102921503.

[4] Bertoletti A, Gehring AJ. The immune response during hepatitis B virus infection. J Gen Virol 2006;87:1439-1449. doi: 10.1099/vir.0.81920-0.

[5] Faure-Dupuy S, Durantel D, Lucifora J. Liver macrophages: Friend or foe during hepatitis B infection? Liver Int 2018;38:1718-1729. doi: 10. 1111/liv.13884.

[6] Webster GJ, Reignat S, Maini MK, Whalley SA, Ogg GS, King A, et al. Incubation phase of acute hepatitis $B$ in man: dynamic of cellular immune mechanisms. Hepatology 2000;32:1117-1124. doi: 10.1053/jhep.2000.19324.

[7] Wieland S, Thimme R, Purcell RH, Chisari FV. Genomic analysis of the host response to hepatitis B virus infection. Proc Natl Acad Sci U S A 2004;101: 6669-6674. doi: 10.1073/pnas.0401771101.

[8] Dunn C, Peppa D, Khanna P, Nebbia G, Jones M, Brendish N, et al. Temporal analysis of early immune responses in patients with acute hepatitis $B$ virus infection. Gastroenterology 2009;137:1289-1300. doi: 10.1053/j.gastro. 2009.06.054.

[9] Chisari FV, Ferrari C. Hepatitis B virus immunopathogenesis. Annu Rev Immunol 1995;13:29-60. doi: 10.1146/annurev.iy.13.040195.000333.

[10] Hagen TM, Huang S, Curnutte J, Fowler P, Martinez V, Wehr CM, et al. Extensive oxidative DNA damage in hepatocytes of transgenic mice with chronic active hepatitis destined to develop hepatocellular carcinoma. Proc Natl Acad Sci U S A 1994;91:12808-12812. doi: 10.1073/pnas.91.26.12808.

[11] Hösel M, Quasdorff M, Wiegmann K, Webb D, Zedler U, Broxtermann M, et al. Not interferon, but interleukin- 6 controls early gene expression in hepatitis $B$ virus infection. Hepatology 2009;50:1773-1782. doi: 10.1002/hep.23226.

[12] Guidotti LG, Rochford R, Chung J, Shapiro M, Purcell R, Chisari FV. Viral clearance without destruction of infected cells during acute HBV infection. Science 1999;284:825-829. doi: 10.1126/science.284.5415.825.

[13] Crispe IN. The liver as a lymphoid organ. Annu Rev Immunol 2009;27: 147-163. doi: 10.1146/annurev.immunol.021908.132629.

[14] Tacke F. Targeting hepatic macrophages to treat liver diseases. J Hepatol 2017;66:1300-1312. doi: 10.1016/j.jhep.2017.02.026.

[15] Naito M, Hasegawa G, Ebe $Y$, Yamamoto T. Differentiation and function of Kupffer cells. Med Electron Microsc 2004;37:16-28. doi: 10.1007/s00795003-0228-x.

[16] Theurl I, Hilgendorf I, Nairz M, Tymoszuk P, Haschka D, Asshoff M, et al. Ondemand erythrocyte disposal and iron recycling requires transient macrophages in the liver. Nat Med 2016;22:945-951. doi: 10.1038/nm.4146.

[17] Martinez FO, Gordon S. The M1 and M2 paradigm of macrophage activation: time for reassessment. F1000Prime Rep 2014;6:13. doi: 10.12703/P6-13.

[18] Murray PJ. Macrophage polarization. Annu Rev Physiol 2017;79:541-566. doi: 10.1146/annurev-physiol-022516-034339.

[19] Vanlandschoot P, Van Houtte F, Roobrouck A, Farhoudi A, Leroux-Roels G. Hepatitis $B$ virus surface antigen suppresses the activation of monocytes through interaction with a serum protein and a monocyte-specific receptor. J Gen Virol 2002;83:1281-1289. doi: 10.1099/0022-1317-83-6-1281.

[20] Neurath AR, Strick N, Sproul P. Search for hepatitis B virus cell receptors reveals binding sites for interleukin 6 on the virus envelope protein. J Exp Med 1992;175:461-469. doi: 10.1084/jem.175.2.461.

[21] Boltjes A, van Montfoort N, Biesta PJ, Op den Brouw ML, Kwekkeboom J, van der Laan LJ, et al. Kupffer cells interact with hepatitis B surface antigen in vivo and in vitro, leading to proinflammatory cytokine production and natural killer cell function. J Infect Dis 2015;211:1268-1278. doi: 10 . 1093/infdis/jiu599.

[22] Vanlandschoot $P$, Van Houtte F, Roobrouck A, Farhoudi A, Stelter F, Peterson $\mathrm{DL}$, et al. LPS-binding protein and CD14-dependent attachment of hepatitis $B$ surface antigen to monocytes is determined by the phospholipid moiety of the particles. J Gen Virol 2002;83:2279-2289. doi: 10.1099/0022-1317-839-2279.

[23] Deng Q, Zhai JW, Michel ML, Zhang J, Qin J, Kong YY, et al. Identification and characterization of peptides that interact with hepatitis $B$ virus via the putative receptor binding site. J Virol 2007;81:4244-4254. doi: 10.1128/JVI. 01270-06.

[24] Li L, Beauchamp MC, Renier G. Peroxisome proliferator-activated receptor alpha and gamma agonists upregulate human macrophage lipoprotein lipase expression. Atherosclerosis 2002;165:101-110. doi: 10.1016/s0021-9150 (02)00203-4.

[25] Makoveichuk E, Castel S, Vilaró S, Olivecrona G. Lipoprotein lipase-dependent binding and uptake of low density lipoproteins by THP-1 monocytes and macrophages: possible involvement of lipid rafts. Biochim Biophys Acta 2004;1686:37-49. doi: 10.1016/j.bbalip.2004.08.015

[26] Ono K, Nishitani C, Mitsuzawa H, Shimizu T, Sano $H$, Suzuki $H$, et al. Mannose-binding lectin augments the uptake of lipid A, Staphylococcus aureus, and Escherichia coli by Kupffer cells through increased cell surface expression of scavenger receptor A. J Immunol 2006;177:5517-5523. doi: 10.4049/jimmunol.177.8.5517.

[27] Op den Brouw ML, Binda RS, Geijtenbeek TB, Janssen HL, Woltman AM. The mannose receptor acts as hepatitis $B$ virus surface antigen receptor 
mediating interaction with intrahepatic dendritic cells. Virology 2009;393: 84-90. doi: 10.1016/j.virol.2009.07.015.

[28] Wang Q, Zhou J, Zhang B, Tian Z, Tang J, Zheng Y, et al. Hepatitis B virus induces IL-23 production in antigen presenting cells and causes liver damage via the IL-23/IL-17 axis. PLoS Pathog 2013;9:e1003410. doi: 10. 1371/journal.ppat.1003410.

[29] Manigold T, Böcker U, Chen J, Gundt J, Traber P, Singer MV, et al. Hepatitis B core antigen is a potent inductor of interleukin-18 in peripheral blood mononuclear cells of healthy controls and patients with hepatitis B infection. J Med Virol 2003;71:31-40. doi: 10.1002/jmv.10445.

[30] Cooper A, Tal G, Lider O, Shaul Y. Cytokine induction by the hepatitis B virus capsid in macrophages is facilitated by membrane heparan sulfate and involves TLR2. J Immunol 2005;175:3165-3176. doi: 10.4049/jimmunol. 175.5.3165.

[31] Iwamoto M, Watashi $\mathrm{K}$, Tsukuda $\mathrm{S}$, Aly $\mathrm{HH}$, Fukasawa M, Fujimoto A, et al. Evaluation and identification of hepatitis B virus entry inhibitors using HepG2 cells overexpressing a membrane transporter NTCP. Biochem Biophys Res Commun 2014;443:808-813. doi: 10.1016/j.bbrc.2013.12.052.

[32] Watashi K, Urban S, Li W, Wakita T. NTCP and beyond: opening the door to unveil hepatitis B virus entry. Int J Mol Sci 2014;15:2892-2905. doi: 10. 3390/ijms15022892.

[33] Oquendo J, Karray S, Galanaud P, Petit MA. Effect of hepatitis B virus on tumour necrosis factor (TNF alpha) gene expression in human THP-1 monocytic and Namalwa B-cell lines. Res Immunol 1997;148:399-409. doi: 10. 1016/s0923-2494(97)82873-8.

[34] Oquendo J, Dubanchet S, Capel F, Mabit H, Petit MA. Suppressive effect of hepatitis $B$ virus on the induction of interleukin- 1 beta and interleukin- 6 gene expression in the THP-1 human monocytic cell line. Eur Cytokine Netw 1996; 7:793-800.

[35] Bouffard P, Lamelin JP, Zoulim F, Pichoud C, Trepo C. Different forms of hepatitis $B$ virus DNA and expression of HBV antigens in peripheral blood mononuclear cells in chronic hepatitis B. J Med Virol 1990;31:312-317. doi: 10. 1002/jmv.1890310413.

[36] Cheng X, Xia Y, Serti E, Block PD, Chung M, Chayama K, et al. Hepatitis B virus evades innate immunity of hepatocytes but activates cytokine production by macrophages. Hepatology 2017;66:1779-1793. doi: 10.1002/hep. 29348.

[37] Zhang JY, Zou ZS, Huang A, Zhang Z, Fu JL, Xu XS, et al. Hyper-activated pro-inflammatory CD16 monocytes correlate with the severity of liver injury and fibrosis in patients with chronic hepatitis B. PLoS One 2011;6:e17484. doi: 10.1371/journal.pone.0017484.

[38] Wu J, Lu M, Meng Z, Trippler M, Broering R, Szczeponek A, et al. Toll-like receptor-mediated control of HBV replication by nonparenchymal liver cells in mice. Hepatology 2007;46:1769-1778. doi: 10.1002/hep.21897.

[39] Zhang E, Lu M. Toll-like receptor (TLR)-mediated innate immune responses in the control of hepatitis B virus (HBV) infection. Med Microbiol Immunol 2015;204:11-20. doi: 10.1007/s00430-014-0370-1.

[40] Isogawa M, Robek MD, Furuichi $Y$, Chisari FV. Toll-like receptor signaling inhibits hepatitis B virus replication in vivo. J Virol 2005;79:7269-7272. doi: $10.1128 /$ JVI.79.11.7269-7272.2005.

[41] Lin Z, Liao W, Ren J. Physicochemical characterization of a polysaccharide fraction from platycladus orientalis (L.) franco and its macrophage immunomodulatory and anti-hepatitis B virus activities. J Agric Food Chem 2016;64: 5813-5823. doi: 10.1021/acs.jafc.6b01387.

[42] Guo F, Han Y, Zhao X, Wang J, Liu F, Xu C, et al. STING agonists induce an innate antiviral immune response against hepatitis $B$ virus. Antimicrob Agents Chemother 2015;59:1273-1281. doi: 10.1128/AAC.04321-14.

[43] Guidotti LG, Borrow P, Hobbs MV, Matzke B, Gresser I, Oldstone MB, et al. Viral cross talk: intracellular inactivation of the hepatitis $B$ virus during an unrelated viral infection of the liver. Proc Natl Acad Sci U S A 1996;93:45894594. doi: $10.1073 /$ pnas.93.10.4589.

[44] Pasquetto V, Guidotti LG, Kakimi K, Tsuji M, Chisari FV. Host-virus interactions during malaria infection in hepatitis $B$ virus transgenic mice. J Exp Med 2000;192:529-536. doi: 10.1084/jem.192.4.529.

[45] Wu J, Meng Z, Jiang M, Pei R, Trippler M, Broering R, et al. Hepatitis B virus suppresses toll-like receptor-mediated innate immune responses in murine parenchymal and nonparenchymal liver cells. Hepatology 2009;49:11321140. doi: 10.1002/hep.22751.

[46] Bradham CA, Plümpe J, Manns MP, Brenner DA, Trautwein C. I. TNF-induced liver injury. Am J Physiol Gastrointest Liver Physiol 1998;275:G387-G392. doi: 10.1152/ajpgi.1998.275.3.G387.

[47] González-Amaro R, García-Monzón C, García-Buey L, Moreno-Otero R, Alonso JL, Yagüe $\mathrm{E}$, et al. Induction of tumor necrosis factor alpha production by human hepatocytes in chronic viral hepatitis. J Exp Med 1994;179:841848. doi: $10.1084 /$ jem.179.3.841.

[48] Sheron N, Lau J, Daniels H, Goka J, Eddleston A, Alexander G], et al. Increased production of tumour necrosis factor alpha in chronic hepatitis $B$ virus infection. J Hepatol 1991;12:241-245. doi: 10.1016/0168-8278(91) 90945-8.
[49] Xu Y, Köck J, Lu Y, Yang D, Lu M, Zhao X. Suppression of hepatitis B virus replication in Tupaia hepatocytes by tumor necrosis factor alpha of Tupaia belangeri. Comp Immunol Microbiol Infect Dis 2011;34:361-368. doi: 10. 1016/j.cimid.2011.05.003.

[50] Cavanaugh VJ, Guidotti LG, Chisari FV. Inhibition of hepatitis B virus replication during adenovirus and cytomegalovirus infections in transgenic mice. J Virol 1998;72:2630-2637. doi: 10.1128/JVI.72.4.2630-2637.1998.

[51] Daniels HM, Meager A, Eddleston AL, Alexander GJ, Williams R. Spontaneous production of tumour necrosis factor alpha and interleukin-1 beta during interferon-alpha treatment of chronic HBV infection. Lancet 1990;335: 875-877. doi: 10.1016/0140-6736(90)90475-k.

[52] Pérez-Alvarez R, Díaz-Lagares C, García-Hernández F, Lopez-Roses L, BritoZerón $\mathrm{P}$, Pérez-de-Lis $\mathrm{M}$, et al. Hepatitis $\mathrm{B}$ virus (HBV) reactivation in patients receiving tumor necrosis factor (TNF)-targeted therapy: analysis of 257 cases. Medicine (Baltimore) 2011;90:359-371. doi: 10.1097/MD. ob013e3182380a76.

[53] Murdaca G, Spanò F, Contatore M, Guastalla A, Penza E, Magnani O, et al. Infection risk associated with anti-TNF- $\alpha$ agents: a review. Expert Opin Drug Saf 2015;14:571-582. doi: 10.1517/14740338.2015.1009036.

[54] French JB, Bonacini M, Ghabril M, Foureau D, Bonkovsky HL. Hepatotoxicity associated with the use of anti-TNF- $\alpha$ agents. Drug Saf 2016;39:199-208. doi: 10.1007/s40264-015-0366-9.

[55] Temel T, Cansu DÜ, Korkmaz C, Kaşifoğlu T, Özakyol A. The long-term effects of anti-TNF- $\alpha$ agents on patients with chronic viral hepatitis C and B infections. Int J Rheum Dis 2015;18:40-45. doi: 10.1111/1756-185X.12467.

[56] Sawhney R, Visvanathan K. Polymorphisms of toll-like receptors and their pathways in viral hepatitis. Antivir Ther 2011;16:443-458. doi: 10 . 3851/IMP1820.

[57] Cavanaugh V], Guidotti LG, Chisari FV. Interleukin-12 inhibits hepatitis B virus replication in transgenic mice. J Virol 1997;71:3236-3243. doi: 10. 1128/JVI.71.4.3236-3243.1997.

[58] Moudi B, Heidari Z, Mahmoudzadeh-Sagheb H, Hashemi M. Gene polymorphisms of macrophage migration inhibitory factor affect susceptibility to chronic hepatitis B virus infection in an Iranian cohort. Microbiol Immunol 2016;60:390-396. doi: 10.1111/1348-0421.12382.

[59] Ebrahim M, Bagheri K, Arababadi MK. Potential roles played by IL- 6 in hepatitis B infection. Future Virology 2014;9:431-438. doi: 10.2217/fvl.14.21.

[60] Tang TJ, Kwekkeboom J, Laman JD, Niesters HG, Zondervan PE, de Man RA, et al. The role of intrahepatic immune effector cells in inflammatory liver injury and viral control during chronic hepatitis B infection. J Viral Hepat 2003;10:159-167. doi: 10.1046/j.1365-2893.2003.00412.x.

[61] Boltjes A, Movita D, Boonstra A, Woltman AM. The role of Kupffer cells in hepatitis B and hepatitis C virus infections. J Hepatol 2014;61:660-671. doi: $10.1016 /$ j.jhep.2014.04.026

[62] Tordjmann T, Soulie A, Guettier C, Schmidt M, Berthou C, Beaugrand M, et al. Perforin and granzyme B lytic protein expression during chronic viral and autoimmune hepatitis. Liver 1998;18:391-397. doi: 10.1111/j.16000676.1998.tb00823.x.

[63] Nakanishi K, Yoshimoto T, Tsutsui H, Okamura H. Interleukin-18 is a unique cytokine that stimulates both Th1 and Th2 responses depending on its cytokine milieu. Cytokine Growth Factor Rev 2001;12:53-72. doi: 10. 1016/s1359-6101(00)00015-0.

[64] Revill P, Yuan Z. New insights into how HBV manipulates the innate immune response to establish acute and persistent infection. Antivir Ther 2013;18: 1-15. doi: 10.3851/IMP2542.

[65] Kimura K, Kakimi K, Wieland S, Guidotti LG, Chisari FV. Interleukin-18 inhibits hepatitis B virus replication in the livers of transgenic mice. J Virol 2002; 76:10702-10707. doi: 10.1128/jvi.76.21.10702-10707.2002.

[66] Nakanishi K, Yoshimoto T, Tsutsui H, Okamura H. Interleukin-18 regulates both Th1 and Th2 responses. Annu Rev Immunol 2001;19:423-474. doi: 10. 1146/annurev.immunol.19.1.423.

[67] Okamura H, Kashiwamura S, Tsutsui H, Yoshimoto T, Nakanishi K. Regulation of interferon-gamma production by IL-12 and IL-18. Curr Opin Immunol 1998;10:259-264. doi: 10.1016/s0952-7915(98)80163-5.

[68] Okamura $\mathrm{H}$, Tsutsui $\mathrm{H}$, Kashiwamura $\mathrm{S}$, Yoshimoto T, Nakanishi K. Interleukin-18: a novel cytokine that augments both innate and acquired immunity. Adv Immunol 1998;70:281-312. doi: 10.1016/s0065-2776(08)60389-2.

[69] Zlotnik A, Yoshie O. Chemokines: a new classification system and their role in immunity. Immunity 2000;12:121-127. doi: 10.1016/s1074-7613(00) 80165-x.

[70] Rossi D, Zlotnik A. The biology of chemokines and their receptors. Annu Rev Immunol 2000;18:217-242. doi: 10.1146/annurev.immunol.18.1.217.

[71] Kakimi K, Lane TE, Chisari FV, Guidotti LG. Cutting edge: Inhibition of hepatitis $B$ virus replication by activated NK T cells does not require inflammatory cell recruitment to the liver. J Immunol 2001;167:6701-6705. doi: 10 . 4049/jimmunol.167.12.6701.

[72] Buonaguro L, Tagliamonte M, Petrizzo A, Damiano E, Tornesello ML, Buonaguro FM. Cellular prognostic markers in hepatocellular carcinoma. Future Oncol 2015;11:1591-1598. doi: 10.2217/fon.15.39. 
[73] Xu L, Yin W, Sun R, Wei H, Tian Z. Kupffer cell-derived IL-10 plays a key role in maintaining humoral immune tolerance in hepatitis $B$ virus-persistent mice. Hepatology 2014;59:443-452. doi: 10.1002/hep.26668.

[74] Sandler NG, Koh C, Roque A, Eccleston JL, Siegel RB, Demino M, et al. Host response to translocated microbial products predicts outcomes of patients with HBV or HCV infection. Gastroenterology 2011;141:1220-1230, 1230. e1-3. doi: 10.1053/j.gastro.2011.06.063.

[75] Li M, Sun R, Xu L, Yin W, Chen Y, Zheng X, et al. Kupffer cells support hepatitis $B$ virus-mediated CD8 + T cell exhaustion via hepatitis $B$ core antigen-TLR2 interactions in mice. J Immunol 2015;195:3100-3109. doi: 10.4049/jimmunol.1500839.

[76] Miyazoe S, Hamasaki K, Nakata K, Kajiya Y, Kitajima K, Nakao K, et al. Influence of interleukin-10 gene promoter polymorphisms on disease progression in patients chronically infected with hepatitis B virus. Am J Gastroenterol 2002;97:2086-2092. doi: 10.1111/j.1572-0241.2002.05926.x.

[77] Taylor AW. Review of the activation of TGF-beta in immunity. J Leukoc Biol 2009;85:29-33. doi: 10.1189/jlb.0708415.

[78] Li H, Zheng HW, Chen $\mathrm{H}$, Xing ZZ, You H, Cong M, et al. Hepatitis B virus particles preferably induce Kupffer cells to produce TGF- $\beta 1$ over pro-inflammatory cytokines. Dig Liver Dis 2012;44:328-333. doi: 10.1016/j.dld.2011. 11.005.

[79] Bility MT, Cheng L, Zhang Z, Luan Y, Li F, Chi L, et al. Hepatitis B virus infection and immunopathogenesis in a humanized mouse model: induction of human-specific liver fibrosis and M2-like macrophages. PLoS Pathog 2014; 10:e1004032. doi: 10.1371/journal.ppat.1004032.

[80] Li TY, Yang Y, Zhou G, Tu ZK. Immune suppression in chronic hepatitis B infection associated liver disease: A review. World J Gastroenterol 2019; 25:3527-3537. doi: 10.3748/wjg.v25.i27.3527.

[81] Chen L, Borozan I, Sun J, Guindi M, Fischer S, Feld J, et al. Cell-type specific gene expression signature in liver underlies response to interferon therapy in chronic hepatitis C infection. Gastroenterology 2010;138:1123-1133.e1-3. doi: $10.1053 /$ j.gastro.2009.10.046.

[82] Zhu Y, Qin B, Xiao C, Lu X, Chen L. Cell-type specific interferon stimulated gene staining in liver underlies response to interferon therapy in chronic HBV infected patients. Dig Dis Sci 2012;57:2355-2361. doi: 10.1007/s10620012-2169-5.

[83] Fleetwood AJ, Dinh H, Cook AD, Hertzog PJ, Hamilton JA. GM-CSF- and MCSF-dependent macrophage phenotypes display differential dependence on type I interferon signaling. J Leukoc Biol 2009;86:411-421. doi: 10 . $1189 / \mathrm{jlb}$.1108702.
[84] Baldanta S, Fernández-Escobar M, Acín-Perez R, Albert M, Camafeita E, Jorge I, et al. ISG15 governs mitochondrial function in macrophages following vaccinia virus infection. PLoS Pathog 2017;13:e1006651. doi: 10. 1371/journal.ppat.1006651.

[85] Yángüez E, García-Culebras A, Frau A, Llompart C, Knobeloch KP, GutierrezErlandsson $\mathrm{S}$, et al. ISG15 regulates peritoneal macrophages functionality against viral infection. PLoS Pathog 2013;9:e1003632. doi: 10. 1371/journal.ppat.1003632.

[86] MacParland SA, Ma XZ, Chen L, Khattar R, Cherepanov V, Selzner M, et al. Lipopolysaccharide and tumor necrosis factor alpha inhibit interferon signaling in hepatocytes by increasing ubiquitin-like protease 18 (USP18) expression. J Virol 2016;90:5549-5560. doi: 10.1128/JVI.02557-15.

[87] Faure-Dupuy S, Delphin M, Aillot L, Dimier L, Lebossé F, Fresquet J, et al. Hepatitis $B$ virus-induced modulation of liver macrophage function promotes hepatocyte infection. J Hepatol 2019;71:1086-1098. doi: 10.1016/j.jhep. 2019.06.032.

[88] Vanlandschoot P, Roobrouck A, Van Houtte F, Leroux-Roels G. Recombinant HBsAg, an apoptotic-like lipoprotein, interferes with the LPS-induced activation of ERK-1/2 and JNK-1/2 in monocytes. Biochem Biophys Res Commun 2002;297:486-491. doi: 10.1016/s0006-291x(02)02243-x.

[89] Cheng J, Imanishi $H$, Morisaki $H$, Liu W, Nakamura $H$, Morisaki T, et al. Recombinant HBsAg inhibits LPS-induced COX-2 expression and IL-18 production by interfering with the NFkappaB pathway in a human monocytic cell line, THP-1. J Hepatol 2005;43:465-471. doi: 10.1016/j.jhep.2005.02.033.

[90] Wang S, Chen Z, Hu C, Qian F, Cheng Y, Wu M, et al. Hepatitis B virus surface antigen selectively inhibits TLR2 ligand-induced IL-12 production in monocytes/macrophages by interfering with JNK activation. J Immunol 2013;190: 5142-5151. doi: 10.4049/jimmunol.1201625.

[91] Yu X, Lan P, Hou X, Han Q, Lu N, Li T, et al. HBV inhibits LPS-induced NLRP3 inflammasome activation and IL-1 $\beta$ production via suppressing the NF- $\mathrm{B} B$ pathway and ROS production. J Hepatol 2017;66:693-702. doi: 10. 1016/j.jhep.2016.12.018.

[92] Tan-Garcia A, Wai LE, Zheng D, Ceccarello E, Jo J, Banu N, et al. Intrahepatic $\mathrm{CD}_{206^{+}}$macrophages contribute to inflammation in advanced viral-related liver disease. J Hepatol 2017;67:490-500. doi: 10.1016/j.jhep.2017.04. 023.

[93] Zang M, Li Y, He H, Ding H, Chen K, Du J, et al. IL-23 production of liver inflammatory macrophages to damaged hepatocytes promotes hepatocellular carcinoma development after chronic hepatitis B virus infection. Biochim Biophys Acta Mol Basis Dis 2018;1864:3759-3770. doi: 10.1016/j.bbadis. 2018.10.004. 